\title{
Chaperonin 60 sustains osteoblast autophagy and counteracts glucocorticoid aggravation of osteoporosis by chaperoning RPTOR
}

Wei-Shiung Lian ${ }^{1,2}$, Jih-Yang Ko ${ }^{3}$, Yu-Shan Chen ${ }^{1,2}$, Huei-Ching Ke ${ }^{1,2}$, Shin-Long Wu ${ }^{1,2}$, Chung-Wen Kuo ${ }^{1,2}$ and Feng-Sheng Wang ${ }^{1,2,4}$

\begin{abstract}
Glucocorticoid excess medication interrupts osteoblast homeostasis and exacerbates bone mass and microstructure loss ramping up the pathogenesis of osteoporotic disorders. Heat shock protein 60 (HSP60) is found to maintain protein function within cellular microenvironment upon encountering detrimental stress. In this study, we revealed that supraphysiological dexamethasone decreased HSP60 expression along with deregulated autophagy in osteoblasts cultures. This chaperonin is required to sustain autophagic markers Atg4, and Atg12 expression, LC3-II conversion, and autophagic puncta formation, and alleviated the glucocorticoid-induced loss of osteogenic gene expression and mineralized matrix accumulation. Regulator-associated protein of mTOR complex 1 (RPTOR) existed in HSP60 immunoprecipitate contributing to the HSP60-promoted autophagy and osteogenesis because knocking down RPTOR impaired autophagic influx and osteogenic activity. HSP60 shielded from RPTOR dysfunction by reducing the glucocorticoid-induced RPTOR de-phosphorylation, aggregation, and ubiquitination. In vivo, forced RPTOR expression attenuated the methylprednisolone-induced loss of osteoblast autophagy, bone mass, and trabecular microstructure in mice. HSP60 transgenic mice displayed increased cortical bone, mineral acquisition, and osteoblast proliferation along with higher osteogenesis of bone marrow mesenchymal cells than those of wild-type mice. HSP60 overexpression retained RPTOR signaling, sustained osteoblast autophagy, and compromised the severity of glucocorticoid-induced bone loss and sparse trabecular histopathology. Taken together, HSP60 is essential to maintain osteoblast autophagy, which facilitates mineralized matrix production. It fends off glucocorticoid-induced osteoblast apoptosis and bone loss by stabilizing RPTOR action to autophagy. This study offers a new insight into the mechanistic by which chaperonin protects against the glucocorticoid-induced osteoblast dysfunction and bone loss.
\end{abstract}

\section{Introduction}

Glucocorticoid medication is widely prescribed to lessen immune irregularity and malignant hematological

\footnotetext{
Correspondence: F.-S. Wang (wangfs@ms33.hinet.net)

${ }^{1}$ Core Laboratory for Phenomics and Diagnostic, Kaohsiung Chang Gung

Memorial Hospital, Kaohsiung, Taiwan

2Department of Medical Research, Kaohsiung Chang Gung Memorial Hospital, Kaohsiung, Taiwan

Full list of author information is available at the end of the article.

Edited by B Zhivotovsky
}

disorders, like allergy, arthritis, and leukemia ${ }^{1}$. Chronic glucocorticoid use damages survival and anabolism of bone cells aggravating excessive bone loss and microarchitecture porosity, prominent deleterious effects notorious for osteoporosis development ${ }^{2,3}$. Organelle deregulation and protein dysfunction speed up osteoblast apoptosis contributing to the progression of glucocorticoid-mediated osteoporosis. For example, high concentrations of glucocorticoid disrupt endoplasmic

\section{(c) The Author(s) 2018}

(c) (i) Open Access This article is licensed under a Creative Commons Attribution 4.0 International License, which permits use, sharing, adaptation, distribution and reproduction c. in any medium or format, as long as you give appropriate credit to the original author(s) and the source, provide a link to the Creative Commons license, and indicate if changes were made. The images or other third party material in this article are included in the article's Creative Commons license, unless indicated otherwise in a credit line to the material. If material is not included in the article's Creative Commons license and your intended use is not permitted by statutory regulation or exceeds the permitted use, you will need to obtain permission directly from the copyright holder. To view a copy of this license, visit http://creativecommons.org/licenses/by/4.0/. 
reticulum $^{4}$ and deteriorates mitochondrial integrity ${ }^{5}$ inducing a burst of reactive oxygen radicals that drastically impedes osteoblast viability. Increased E3 ubiquitin ligase signaling ramps up glucocorticoid-induced osteopenia in mice 6 . The mechanism underlying the glucocorticoid-impaired intracellular homeostasis and organelle machinery in osteoblasts upon glucocorticoid stress has not been well investigated.

Autophagy is a sophisticated intracellular program, including progressive autophagosome formation and lysosomal degradation, responsible for removing abandoned organelle or proteins to maintain regular physiological activity of cells upon experiencing adverse conditions ${ }^{7}$. As regards the biological role of autophagy in skeletal metabolism, knockout mice deficient in autophagic vesicle builder Atg5 in osteoblasts show poor mineralization capacity and low bone mineral density $^{8}$. Mice lacking Atg5 and Atg7 in chondrocytes exhibit bone undergrowth ${ }^{9}$. Poor autophagy slows down bone development in fibroblast growth factor knockout mice ${ }^{10}$. In addition, mice lacking upstream autophagy regulator mammalian target of rapamycin complex 1 (mTORC1) in osteoblasts display low bone mass and meager trabecular microstructure ${ }^{11}$, whereas osteoclastspecific mTORC1 knockout mice reveal high bone mineral density owing to impaired osteoclastic resorption capacity ${ }^{12}$. The role. of mTORC1 signaling components in the progression of glucocorticoid-induced osteoporosis remains elusive.

Heat shock protein 60 (HSP60) is a mitochondrial chaperonin, stabilizing protein function through chaperoning unfolded proteins into a folded state ${ }^{13}$. HSP60 knockout causes intestinal epithelial stem cells to lose stemness and proliferation capacity ${ }^{14}$. Decreased HSP60 impairs mitochondrial integrity worsening the fused sarcoma-associated neurodegenerative disorders ${ }^{15}$, whereas increasing this chaperonin promotes mitochondrial energy expenditure of murine skeletal muscle during endurance training ${ }^{16}$. Upregulating HSP60 facilitates alveolar macrophage autophagy to augment inflammatory responses in lung tissue upon ischemia and reperfusion ${ }^{17}$. We previously found that mice overexpressing HSP60 exhibited minor responses to chondrocyte apoptosis and knee osteoarthritis progression ${ }^{18}$. Silencing HSP60 expression induced bone loss in rats ${ }^{19}$. Given that HSP60 participated in bone metabolism, we hypothesized that it may regulate osteoblast autophagy during the pathogenesis of glucocorticoid excess-induced osteoporotic skeletons.

This study is undertaken to investigate molecular events underlying HSP60 action to glucocorticoid-impaired osteoblast autophagy and osteogenic differentiation, and utilized HSP60 transgenic mice to verify its regulatory functions to osteoblast autophagy, survival, and bone mass homeostasis in long-term glucocorticoid excesstreated skeletal tissue.

\section{Results \\ HSP60 loss worsened glucocorticoid-inhibited autophagy and osteogenesis}

To examine whether HSP60 affected autophagy in glucocorticoid-stressed osteoblasts, cell cultures were incubated in osteogenic medium containing $1 \mu \mathrm{M}$ dexamethasone, an in vitro model that mimics glucocorticoid excess-induced bone loss ${ }^{20}$. Dexamethasone significantly reduced HSP60 mRNA expression and protein levels along with slight HSP60 immunofluorescence in cytoplasmic compartment as compared with vehicle group (Fig. 1a). Forced HSP60 expression (Fig. 1b) lessened the glucocorticoid-induced loss of osteogenic activities, like osteocalcin expression (Fig. 1c) and von Kossa stainingpositive mineralized matrix formation (Fig. 1d), whereas knocking down HSP60 significantly reduced baseline osteogenesis as compared with scrambled controls (Fig. 1b, c, d).

With regard to autophagic activity, glucocorticoid or HSP60 interference significantly decreased autophagy markers Atg4, and Atg12 expressions (Fig. 1e), concomitant with reduced LC3-II levels, LC3-II/LC3-I ratio (Fig. 1f), and autophagic puncta as evident from fluorescent probe monodansylcadaverin for autophagic vacuoles (Fig. 1g). Increasing HSP60 attenuated the glucocorticoid-mediated loss of autophagic marker expressions, LC3-II abundances, LC3-II/LC3-I ratio, and autophagic vesicles (Fig. 1e, f, g), which was suggestive of HSP60 maintaining autophagic influx in osteoblasts upon glucocorticoid stress.

\section{HSP60 regulated RPTOR signaling}

To understand how HSP60 retained autophagy in glucocorticoid-stressed osteoblasts, HSP60 immunoprecipitates were isolated for electrophoresis. Sodium dodecyl sulfate-polyacrylamide gel electrophoresis analysis revealed that a protein corresponding to $\sim 150$ $\mathrm{kDa}$ was decreased in the glucocorticoid-treated cells (Fig. 2a). The protein band of interest was cut for in-gel trypsin digestion and liquid chromatography to characterize peptide sequence. The protein was highly homologous to regulatory-associated protein of mTOR (RPTOR) as evident from tandem mass spectrum (Fig. 2b, c). Consistently, glucocorticoid-treated osteoblasts showed weak HSP60 (green) and RPTOR (red) immunofluorescence (Fig. 2d). In addition, glucocorticoid or HSP60 RNAi treatment significantly depleted RPTOR expressions. Forced HSP60 expression lessened the extent of glucocorticoid-induced RPTOR loss (Fig. 2e). Proximity ligation analyses also confirmed an interaction between HSP60 and RPTOR as evident 


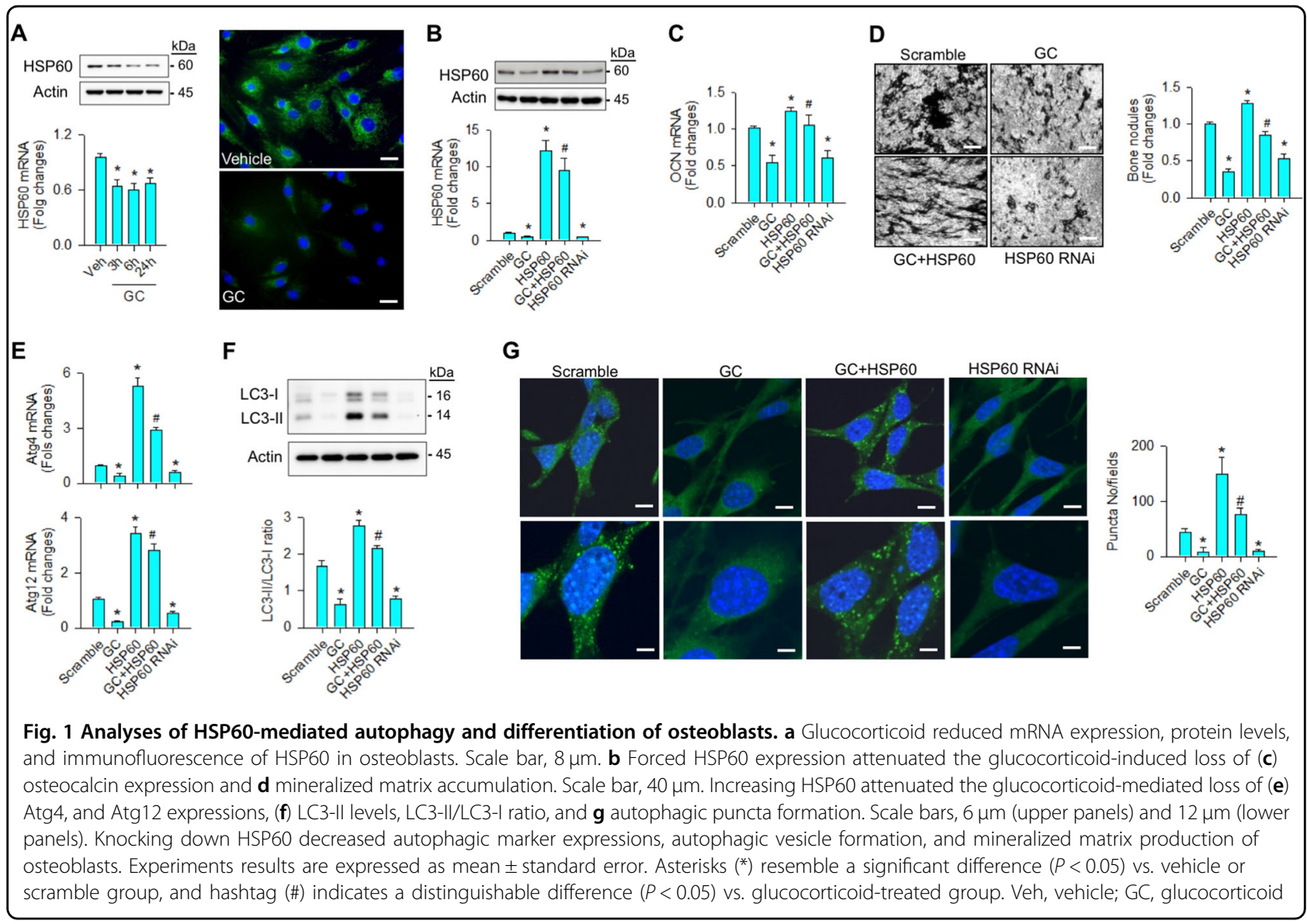

from abundant protein ligated complexes displaying fluorescent spots in the scramble control-transfected and HSP60-transfected cells, whereas glucocorticoid stress or HSP60 interference inhibited the reaction (Fig. 2f).

\section{RPTOR attenuated the glucocorticoid suppression of autophagy and osteogenesis}

To test whether RPTOR signaling changed glucocorticoid-inhibited autophagy or osteogenesis, cell cultures were transfected with RPTOR complementary DNA (cDNA) or RNA interference (RNAi). Gain of RPTOR function (Fig. 3a) attenuated glucocorticoidinhibited Atg4, and Atg12 expressions, LC3-II concentrations, LC3-II/LC3-I ratio (Fig. 3b), and autophagic puncta formation (Fig. 3c). Interfering with RPTOR significantly decreased baseline expressions of autophagic marker along with very few autophagosome vesicles (Fig. 3a, b, c). In addition, forced RPTOR expression alleviated the glucocorticoid-induced loss of osteogenic markers Runx2 and osteocalcin expressions (Fig. 3d), and mineralized nodules accumulation (Fig. 3e). Knocking down RPTOR also significantly downregulated baseline osteogenic activities (Fig. 3d, e).

\section{HSP60 ameliorated glucocorticoid-induced loss of RPTOR stability}

We examined whether HSP60 affected RPTOR stability because it is found to modulate protein function through post-translational modification ${ }^{21,22}$. Glucocorticoid or HSP60 RNAi decreased levels of phosphorylated ERK, phosphorylated Ser473-Akt, and phosphorylated RPTOR (Fig. 4a), whereas concentrations of Bax, cytochrome c, and cleaved caspase 3 were increased (Fig. 4b). Increasing HSP60 retained abundances of these three phosphorylated proteins and mitigated the glucocorticoidupregulated apoptotic signaling (Fig. 4a, b).

Given that unstable proteins are inclined to form aggregates $^{21}$, we examined whether glucocorticoid or HSP60 affected protein aggregation. To prepare positive controls, acetone was added to cell lysate to induce protein aggregation. Aggregated proteins in cell lysates were ultra-centrifuged and electrophoresed. Glucocorticoid or HSP60 knockdown increased levels of aggregated proteins. Increased RPTOR also existed in the aggregated proteins (Fig. 4c). With ubiquitin antibody probing, glucocorticoid, or HSP60 RNAi upregulated concentrations of ubiquitinated proteins (Fig. 4d) and ubiquitinated RPTOR (Fig. 4e). Increasing HSP60 expression attenuated 


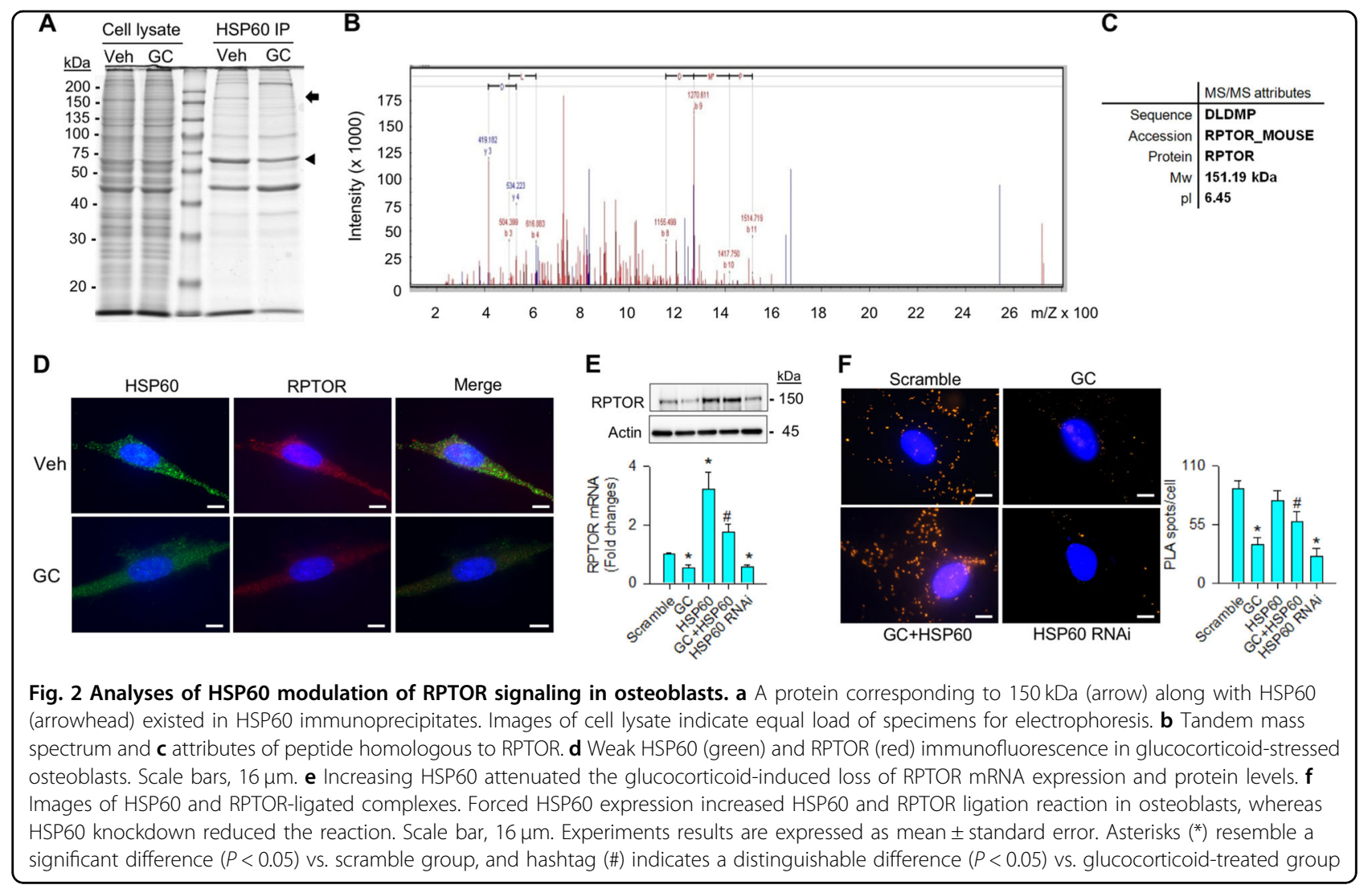

glucocorticoid-enhanced levels of aggregated and ubiquitinated proteins along with reduced abundances of aggregated and ubiquitinated RPTOR (Fig. 4c, d, e). We tested if inhibiting RPTOR ubiquitination affected autophagy. Inactivating ubiquitin by proteasome inhibitor MG132 attenuated levels of ubiquitinated RPTOR and lessened the glucocorticoid-induced loss of RPTOR, LC3II levels, and LC3-II/LC3-I ratio (Fig. 4f). Furthermore, glucocorticoid or HSP60 RNAi or RPTOR RNAi significantly increased osteoblasts apoptosis compared with scrambled controls as evident from fluorescent TUNEL staining, whereas overexpressing HSP60 or RPTOR attenuated glucocorticoid-induced apoptosis (Fig. 4g).

\section{RPTOR alleviated glucocorticoid-induced bone deterioration}

Given that RPTOR signaling promoted osteoblast function in vitro, we verified whether it affected glucocorticoid-induced bone loss. Mice were injected with lentivirus RPTOR and followed by methylprednisolone treatment for 4 consecutive weeks (Fig. 5a). It attenuated the glucocorticoid-induced loss of RPTOR levels in bone tissue (Fig. 5b). Glucocorticoid-induced sparse trabecular microstructure (Fig. 5c) and significantly decreased bone mineral density (BMD), trabecular bone volume (BV/TV), trabecular number (Tb.N), and trabecular thickness (Tb.Th) (Fig. 5d). Increasing RPTOR lessened the severity of glucocorticoid-mediated loss of bone mass, microarchitecture, and morphometric characteristics (Fig. 5c, d). It also retained autophagy markers Atg4, Atg12, LC3-II levels, and LC3-II/LC3-I ratio (Fig. 5e), and preserved LC3 immunostaining in osteoblasts along trabecular bone in glucocorticoid-treated skeletons (Fig. 5f).

\section{HSP60 overexpression attenuated the glucocorticoid- induced bone loss}

To understand whether increasing HSP60 changed glucocorticoid-induced bone loss, mice that overexpressed HSP60 (HSP60Tg) driven by PGK promoter were bred for study ${ }^{18}$. HSP60Tg mice carried the $1.7 \mathrm{kbp}$ construct of interest; HSP60 expression was significantly increased in bone tissue (Fig. 6a). They gained body weight comparable to wild-type mice throughout the study (Fig. 6b). With respect to skeletal phenotypes, HSP60 overexpression significantly increased bone length and bone weight along with thicker cortical bone as compared with wild-type mice (Fig. 6c). Bone mineral acquisition and osteoblast growth were also promoted in HSP60Tg mice as evident from fluorescent calcein labeling (Fig. 6d) and proliferating cell nuclear antigen immunostaining (Fig. 6e), respectively. Of interest, 
A

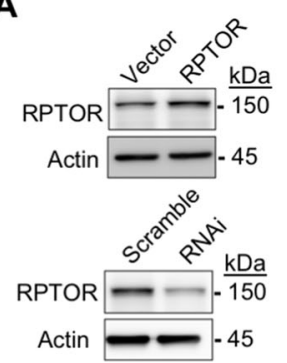

C
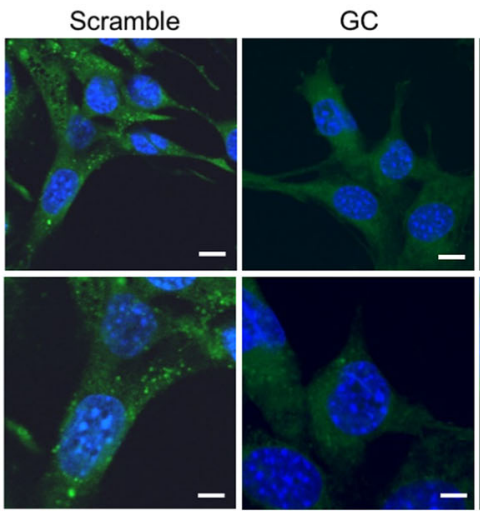

D

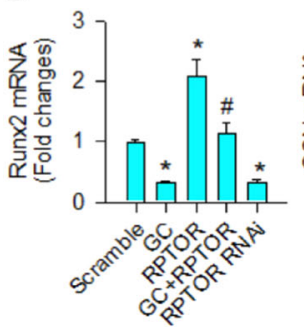

GC

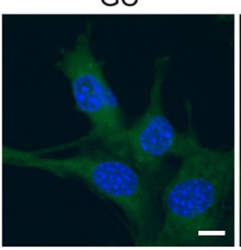

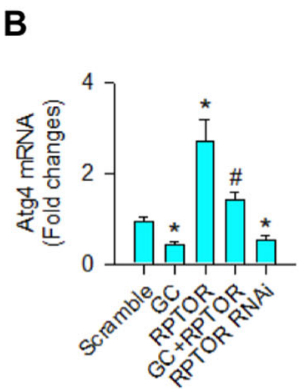
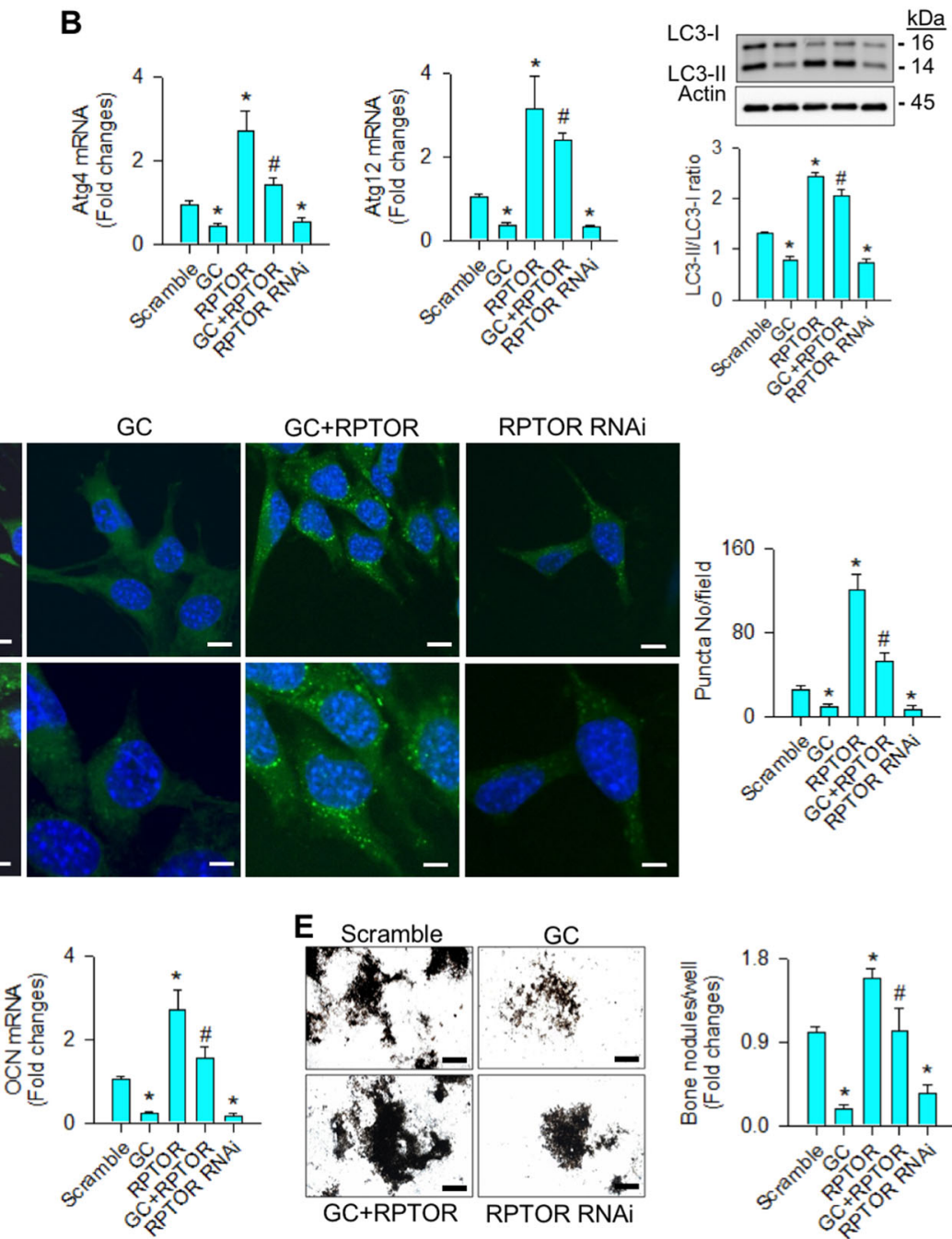

Fig. 3 RPTOR promoted autophagy and osteogenic activities. a RPTOR levels in RPTOR cDNA- and RNAi-transfected osteoblasts. b Increasing RPTOR alleviated the glucocorticoid-induced loss of Atg4, and Atg12 expressions, LC3-II levels, LC3-II/LC3-I ratio, and c autophagic puncta. Scale bars, $8 \mu \mathrm{m}$ (upper panels) and $16 \mu \mathrm{m}$ (lower panels). It also restored $\mathbf{d}$ Runx2 and osteocalcin expression, and e mineralized nodule formation. Knocking down RPTOR impaired autophagic marker expression, autophagic vesicle formation, and osteogenic activity. Scale bar, 46 um. Experiments results are expressed as mean \pm standard error. Asterisks $\left(^{*}\right)$ resemble a significant difference $(P<0.05)$ vs. scramble group, and hashtag $(\#)$ indicates a distinguishable difference $(P<0.05)$ vs. glucocorticoid-treated group

abundant trabecular bone microstructure remained in HSP60Tg mice at 4 weeks after methylprednisolone stress, whereas decreased trabecular architecture existed in methylprednisolone-treated wild-type mice (Fig. 6f). Consistently, the severity of glucocorticoid-induced loss of morphometric characteristics BMD, BV/TV, Tb.Th, and Tb.N was significantly compromised in HSP60Tg mice (Fig. 6g).

\section{HSP60 reduced glucocorticoid-mediated loss of autophagy and osteogenesis}

We verified whether HSP60 overexpression changed bone histology or osteoblast autophagy in skeletal tissue at 4 weeks after methylprednisolone treatment.
Glucocorticoid escalated trabecular bone loss histopathology and significantly decreased BV/TV in wild-type mice. These adverse effects were significantly attenuated in HSP60Tg mice (Fig. 7a). Furthermore, HSP60 overexpression retained Atg4, and Atg12 expression (Fig. 7b), and LC3-II concentration along with increased LC3-II/ LC3-I ratio (Fig. 7c) but decreased Bax and cleaved caspase3 levels (Fig. 7d). It also alleviated the glucocorticoidinduced loss of LC3 immunostaining in osteoblasts (Fig. 7e).

To verify whether HSP60 changed osteogenic differentiation capacity, primary bone marrow mesenchymal cells were isolated and incubated in osteogenic medium. In the wild-type group, glucocorticoid significantly 


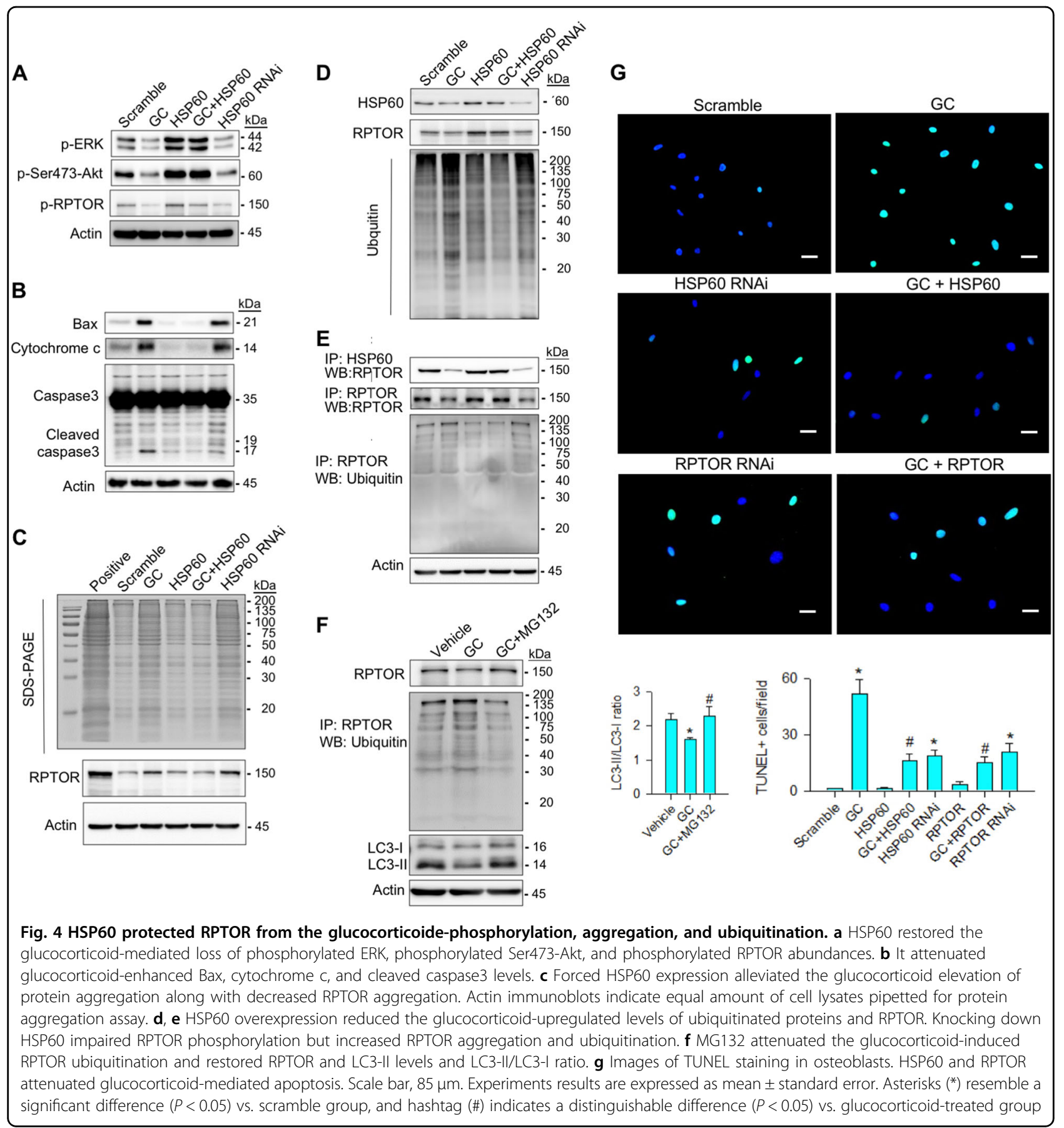

inhibited Runx2, collagen $1 \alpha 1$, and osteocalcin expressions (Fig. 7f), and mineralized matrix synthesis (Fig. 7g). The extent of glucocorticoid-inhibited osteogenesis was significantly lessened in HSP60Tg mice (Fig. 7f, g).

\section{Discussion}

Autophagy is involved in disposal of unwanted molecules to harmonize intracellular microenvironment, enabling cells to overcome deleterious conditions ${ }^{7}$.
Deregulated autophagy ratchets up the pathogenesis of skeletal and arthritic disorders. For example, low autophagic breakdown of misfolded collagen worsens osteogenesis imperfecta in mice ${ }^{23}$. Overacted autophagy mediates metal implant particle-induced osteolysis ${ }^{24}$. Proteasome inhibitor bortezomib-suppressed autophagy weakens bone metastasis capacity of multiple myeloma cells $^{25}$. Poor osteoblast autophagy of crystal urate increases chronic gout development ${ }^{26}$. Aberrant 


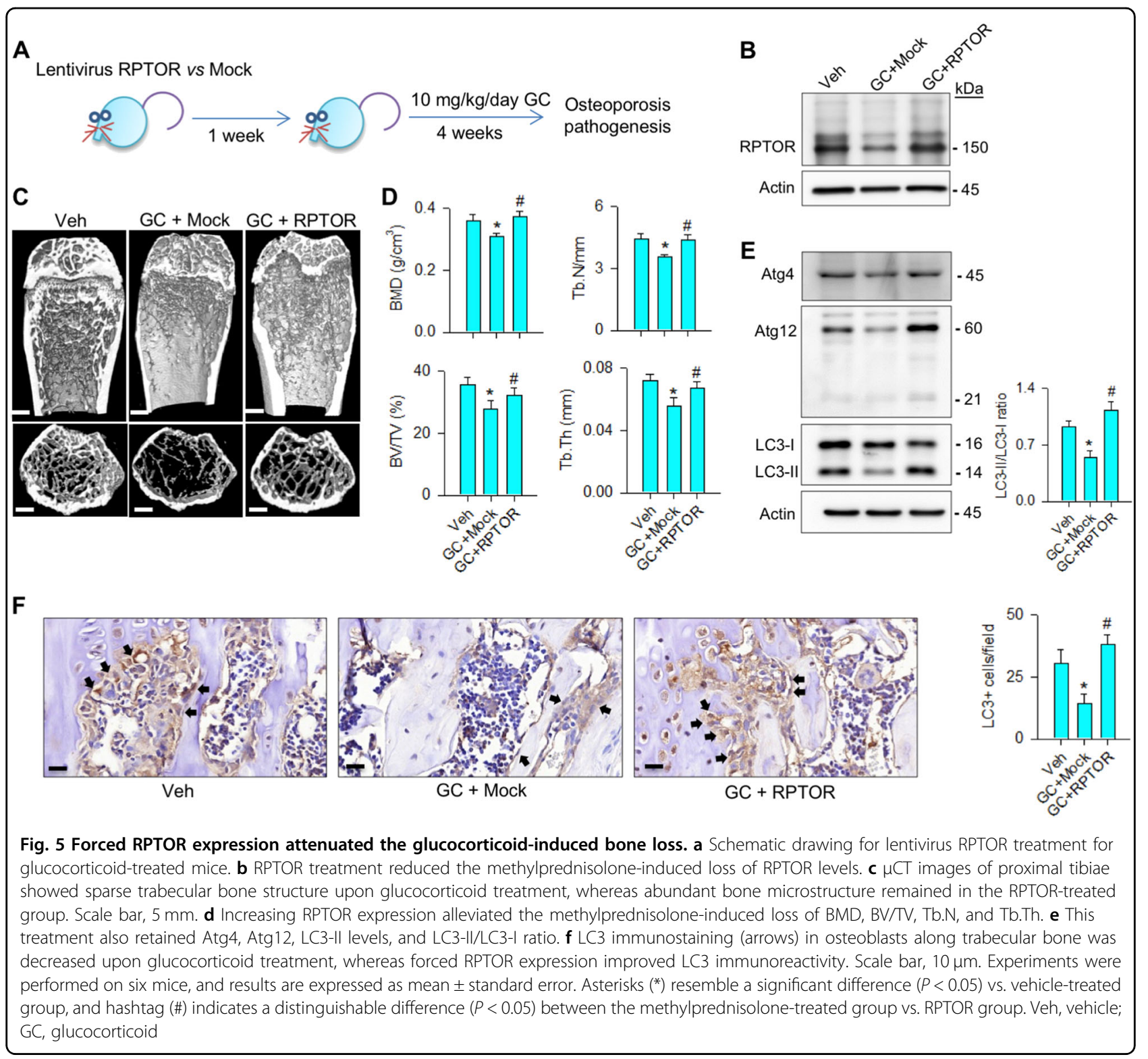

autophagy hinders osteogenesis of bone marrow mesenchymal stem cells worsening diabetes-induced bone mass $\operatorname{loss}^{27}$. The contribution of osteoblast autophagy to glucocorticoid-induced excessive bone catabolism has not been well defined. This study is the first uncovering HSP60 action indispensable in osteoblast autophagy for protecting skeletal tissue from glucocorticoidinduced osteoporosis. It also throws light on new mechanistic events by which mitochondrial chaperonin pathway preserves RPTOR function to autophagic influx within osteoblast microcompartment facilitating bone tissue homeostasis and microstructure integrity.

Analyses of osteoblast culture models revealed that HSP60 was required to osteogenesis because silencing this mitochondrial chaperonin largely led to mineralized matrix synthesis below baseline, overexpressing it enabled cell cultures to uphold osteogenic differentiation capacity upon supraphysiological glucocorticoid-mediated adverse stress. Increasing evidence reveals that high dose of therapeutic glucocorticoid disturbs mitochondrial machinery provoking cell damage or tissue deterioration, like skeletal muscle wasting ${ }^{28}$, necroptosis of acute lymphoblastic leukemic cells ${ }^{29}$, and fat overproduction of adipose tissue ${ }^{30}$. Loss of HSP60 function also perturbs extracellular matrix metabolism and amplifies apoptotic programs $^{31,32}$. This study showed that HSP60 maintained autophagic influx beneficial for fending off glucocorticoid-induced apoptosis and mineralized matrix underproduction, indicating that glucocorticoid stress cutoff a fine-tuned organelle crosstalk between 


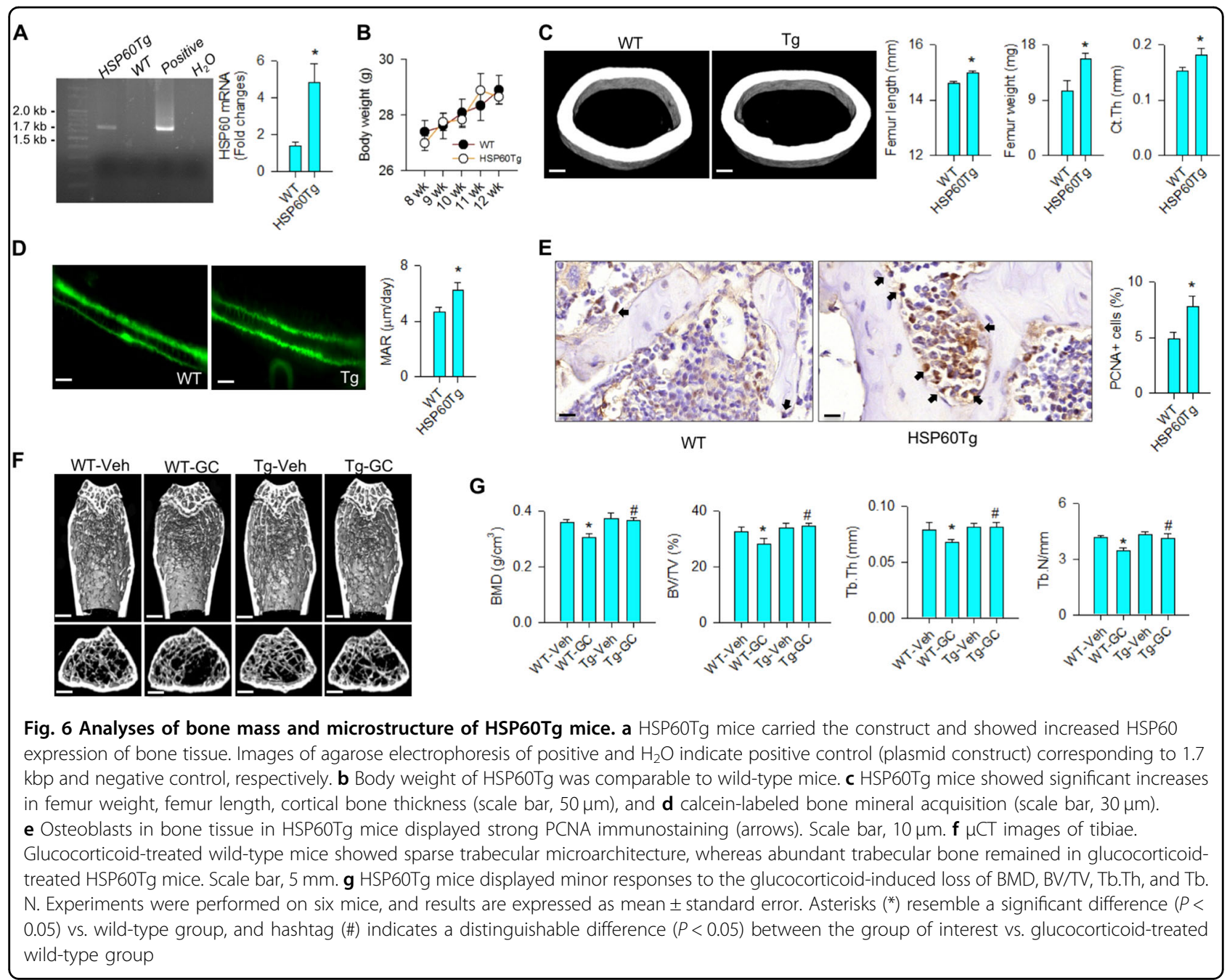

autophagosome and mitochondria aggravating osteoblast dysfunction. These interesting findings prompted us to decipher the molecular event by which HSP60 regulated osteoblast autophagy.

Of note, arrays of analyses uncovered that RPTOR interplayed with HSP60 protecting osteoblasts against the glucocorticoid-impaired autophagy and differentiation. The regulatory action of RPTOR to skeletal tissue metabolism and osteoporosis pathogenesis seems like inconclusive. For example, bone marrow mesenchymal stem cells from RPTOR knockout mice favorably turn out to be osteogenic lineages ${ }^{33}$. On the contrary, bone tissuespecific RPTOR knockout mice show bone underdevelopment $^{34}$ and poor osteogenic activity of preosteoblasts $^{11}$. Activation of mTORC1 prevents from osteoporosis progression in mice overexpressing Wnt1 in osteocytes $^{35}$. We uncovered its role in preserving osteoblast function and bone integrity because increasing RPTOR alleviated the severity of glucocorticoid-induced bone mass loss and microstructure deterioration.
Together with our findings and other groups' investigations, this molecule appeared to serve different functions depending on bone cell types and deleterious stresses impacted on skeletal tissue. Given that HSP60 interacted with RPTOR, further experiments were performed to delineate in detail how HSP60 warded off the glucocorticoid-induced RPTOR loss.

Profound results of immunoprecipitation analysis revealed that HSP60 sustained RPTOR function by controlling multiple post-translational modification reactions of RPTOR, like phosphorylation, aggregation, and ubiquitination. HSP60 is found to convert mitochondrial proteins into a folding status, which drives them away from aggregation ${ }^{36,37}$. It also affects kinase phosphorylation $^{38}$ and cellular protein degradation ${ }^{39}$. In this study, we conveyed a new insight into chaperone machinery protective against the glucocorticoid-mediated RPTOR dysfunction. In addition to RPTOR stability, analyses of increased survival regulators ERK and Akt, and decreased pro-apoptotic signaling Bax and cytochrome c release 


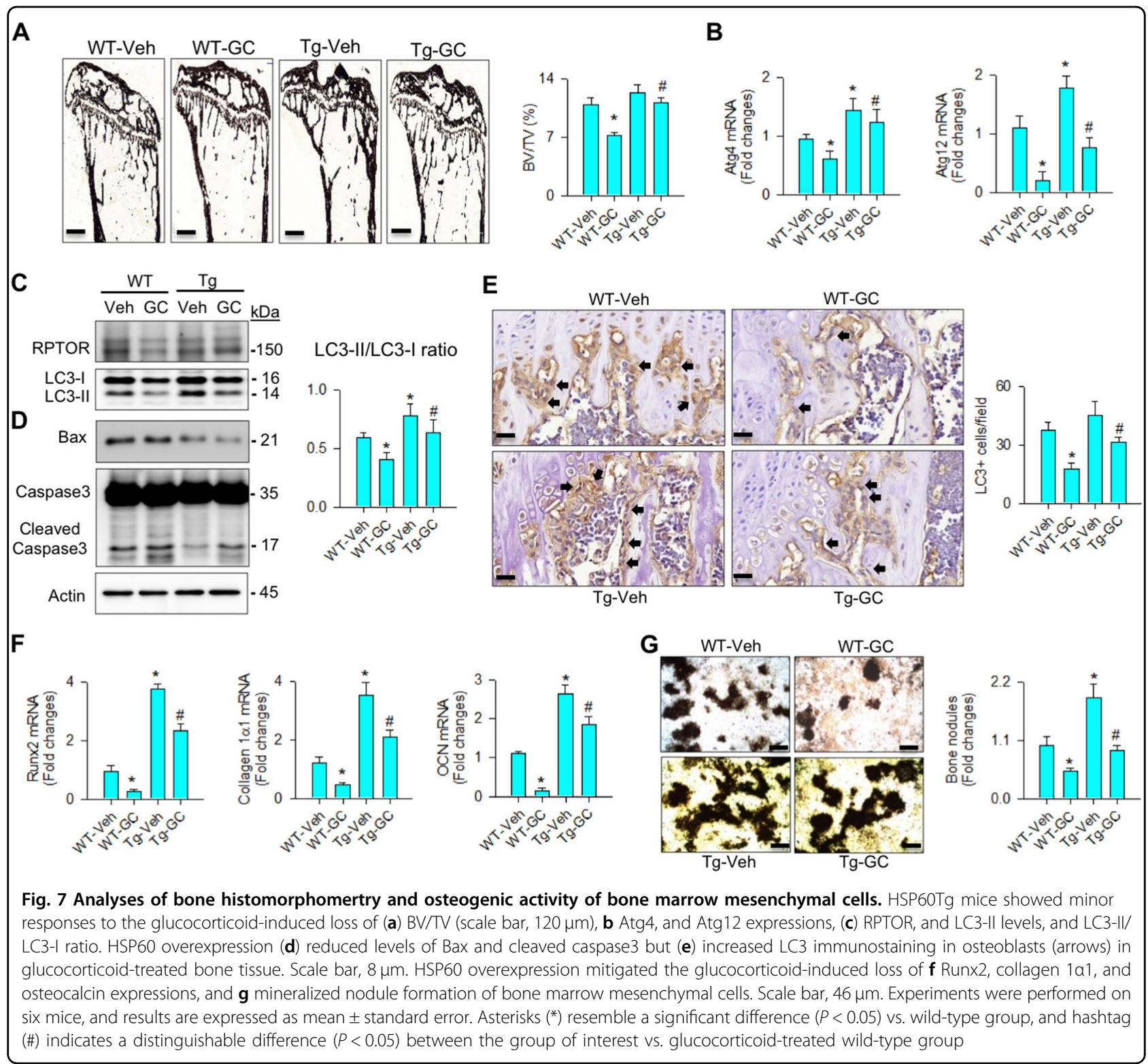

consolidated the experimental findings showing that HSP60 facilitated osteoblast survival. Investigations were in agreement with other groups' studies demonstrating that Akt and ERK pathways phosphorylate RPTOR facilitating mTOR1 activity and cell growth ${ }^{40,41}$. Although it warrants further analysis in the future to characterize how chaperonin activates these regulators, changes in various signaling pathways indicated that multiple reactions within intracellular environment participated in stabilizing RPTOR to curtail the glucocorticoid excess-induced loss of osteogenic activity.

Intriguing findings were that HSP60Tg mice showed moderate bone overgrowth phenotypes like thickened cortical bone and increased bone mineral accretion, concomitant with intensive osteoblast proliferation. These anabolic effects kept skeletal tissue away from methylprednisolone excess-induced bone mineral density loss and trabecular porosity histopathology. Consistent with the analyses of in vitro osteoblast models, overexpressing HSP60 improved RPTOR signaling and autophagy to delay glucocorticoid-mediated osteoblast dysfunction. Collective investigations of sustained osteoblast autophagy and high osteogenic differentiation of bone marrow mesenchymal cells in HSP60Tg mice underpinned the bone anabolic function of HSP60 able to drag the glucocorticoid excess-mediated bone deterioration.

Limitation of this study is that other regulators existing in HSP60 immunoprecipitate may directly or indirectly modulate osteoblast autophagy to alter bone formation upon glucocorticoid stress. It also indicates that HSP60 


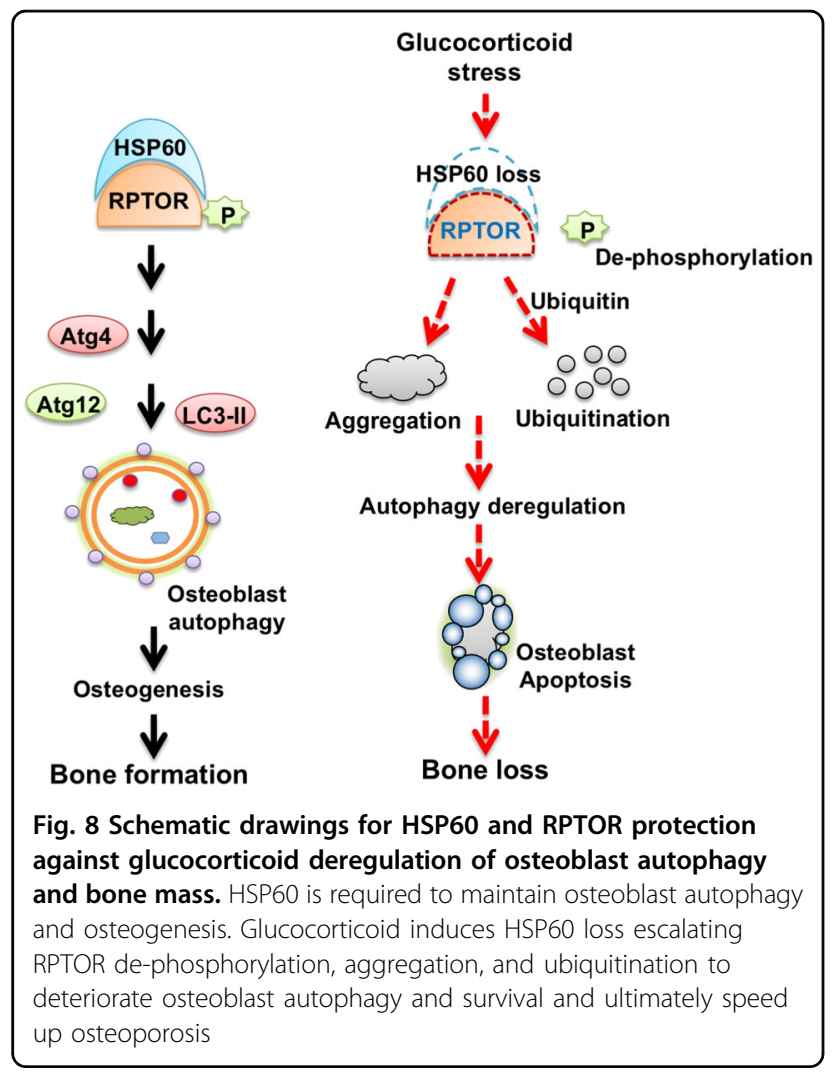

plays an important role in dealing with the complex nature of intracellular organelle intercommunication during glucocorticoid-deregulated osteoblast behavior. Taken together, plausible investigations revealed that HSP60 is required to osteoblast survival and mineralized matrix anabolism compromising the development of glucocorticoid-mediated osteoporotic skeletons through sustaining RPTOR function to osteoblast autophagy as schemed in Fig. 8. This study highlights an emerging molecular mechanism by which chaperone HSP60 harmonizes organelle homeostasis within osteoblasts delaying the development of osteoporosis.

\section{Methods}

\section{Glucocorticoid-stressed osteoblast cultures}

Murine MC3T3-E1 osteoblasts were maintained in Dulbecco's Modified Eagle's Medium (DMEM) and 10\% fetal bovine serum. $1 \times 10^{5}$ cells/well were incubated in 24-well plates containing osteogenic medium (DMEM, 10\% fetal bovine serum, $10 \mathrm{mM} \beta$-glycerophosphate, and $50 \mu \mathrm{g} / \mathrm{ml}$ ascorbic acid) (R\&D System) with $1 \mu \mathrm{M}$ dexamethasone or vehicle dimethyl sulfoxide for 18 days. Medium was changed every 3 days. Mineralized matrix deposits in cell cultures were detected using von Kossa staining, and area of mineralized nodules in three random fields in each well was measured using Zeiss Inverted microscopy and image analysis software, as previously described ${ }^{42}$.

\section{RNA interference and CDNA transfection}

cDNA coded human HSP60 or RPTOR were constructed into vectors pUSEamp $(+)$ ) (Upstate Biotechnolog, Lake Placid, NY). RNA interference targeting HSP60 (\#11067) and RPTOR (\#178055) were obtained from Applied Biosystems Biotechnology (ThermoFischer Scientific). Aliquots of $2 \mu \mathrm{g}$ cDNA, RNAi or scramble controls were mixed with Lipofectamine ${ }^{\mathrm{TM}} 3000$ Transfection Reagent (Invitrogen ${ }^{\mathrm{TM}}$; ThermoFischer Scientific) and transferred into cell cultures, according to the manufacturer's instructions.

\section{Immunofluorescence analysis of HSP60, RPTOR, and autophagic puncta}

After removing culture medium and rinsing with phosphate-buffered saline (PBS), cell cultures were fixed with 4\% formaldehyde in PBS and blocked with normal goat serum (Cell Signaling Technology, Danvers, MA). HSP60 and RTPOR immunoreactivity in cell cultures were probed using HSP60 (\#ab3080, Abcam Cambridge, MA) and RPTOR antibodies (\#2280, Cell Signaling Technology, Danvers, MA). Goat anti-mouse IgG conjugated Alexa Fluor 488 and donkey anti-mouse IgG conjugated Alexa Fluor 675 (Abcam, Cambridge, MA) were used as secondary antibodies. After counterstaining with 4',6-diamidino-2-phenylindole (DAPI) and covering with Prolong Gold Antifade Reagent (Cell Signaling Technology, Danvers, MA), immunofluorescence was evaluated using an Olympus Laser Confocal Microscope system. In some experiments, autophagic puncta in cell cultures were detected using fluorescent monodansylcadaverin probe of Autophagy Detection Kits (Abcam, Cambridge, MA), according to the manufacturer's instructions. Number of puncta in 30 cells in three random fields of each well were counted.

\section{RT-quantitative PCR analysis of mRNA expression}

Cell cultures were mixed with Trizol reagent to extract total RNA. Reverse transcription of $1 \mu \mathrm{g}$ total RNA was performed using ReadScript ${ }^{\oplus}$ Two-Step cDNA Synthesis Kits (Sigma-Aldrich). For PCR analysis, $2 \times$ TaqMan $^{\circ}$ Universal PCR Master Mix along with primers for probing HSP60 (forward, 5'-CGTTGCCAATAACACAAACG-3'; reverse, 5'-CGTTGCCAATAACACAAACG-3'), RPTOR (forward, 5'-GCAGAGCTGGAGAATGAAGG-3'; reverse, 5'-GTCGAGGCTCTGCTTGTA CC-3'), Atg4 (forward, 5'-CCAGCTTCAGCAAGATCTCC-3'; reverse, 5'-ATA CATCCCCAGCCACAGTC-3'), Atg12 (forward, 5'-CCA GCCCAATAGGACTC TTTAAC-3'; reverse, 5' -CACA GCACCGAAATGTCTC-3'), Runx2 (forward, 5'-CCAG CAGCACTCCATATCTC-3'; reverse, 5'-CAGCGTCAA CACCATCATTC $-3^{\prime}$ ), collagen $1 \alpha 1$ (forward, 5'-CAC CCTCAAGAGCCTGAGTC-3'; reverse, 5' - CA GACGG CTGAGTAGGGAAC-3'), osteocalcin (forward, 5'-CAA 
GCAGGGAGGCA ATAAGG-3'; reverse, 5'-CGTCAC AAGCAGGGTTAAGC-3'), and actin (forward, 5'-GAC GGCCAGGTCATCACTAT-3'; reverse: $5^{\prime}$-CTTCTGC ATCCTGTCAGCA A-3') were mixed with the reverse transcription products. Gradient thermal programs and threshold values $(\mathrm{Ct})$ computation were performed using a ABI 7900 Detection System (Applied Biosystems). Expressions of mRNA were calculated using the equation $2^{-\Delta \Delta \mathrm{Ct}}$, where $\Delta \mathrm{Ct}$ resembled the difference in $\mathrm{Ct}$ values between the gene of interest and actin, and $\Delta \Delta \mathrm{Ct}$ stood for the difference in $\Delta \mathrm{Ct}$ between the glucocorticoid and vehicle group.

\section{Assessment of immunoblotting, protein aggregation, and immunoprecipitation}

Lysates of $5 \times 10^{6}$ cells were prepared using Mammalian Cell Extraction Kits (Abcam, Cambridge, MA). HSP60, RPTOR, Atg4 (\#ab108322, Abcam, Cambridge, MA), Atg12 (sc-68884, Santa Cruz Biotechnology), LC3 (\#PA1-16930, ThermoFischer Scientific), phosphorylated ERK (\#9106), phosphorylated Ser473-Akt (\#9721), phosphorylated RPTOR (\#2083), Bax (\#2774), cytochrome c (\#12963), caspase3 (\#9662), ubiquitin (\#3936), and actin (\#4967, Cell Signaling Technology, Danvers, MA) antibodies were used for probing the designated proteins in lysates using immunoblotting protocols. In some experiments, protein aggregates in $100 \mu \mathrm{g}$ cell lysates were isolated after ultrahigh centrifugation at $10,000 \mathrm{~g}$ at $4{ }^{\circ} \mathrm{C}$ for $30 \mathrm{~min}$, as previously described $^{20}$. For positive controls, $100 \mu$ g cell lysates were mixed with equal volume of ice-cold acetone. Pellets were separated by electrophoresis and stained using Coomassie blue, and RPTOR levels in the pellet was probed using immunoblotting protocols. In addition, HSP60 and RPTOR immunoprecipitates were isolated for immunoblotting probed using ubiquitin antibody.

\section{Liquid chromatography and tandem mass spectrometry}

Aliquots of HSP60 immunoprecipitates were electrophoresed, and protein bands of interest in the gel were cut for trypsin hydrolysis, as previously described ${ }^{19}$. Peptides in the hydrolyte were eluted through an Acclaim PepMap 100 column using a high-performance liquid chromatography system (Dionex Ultimate 3000, Dionex Corp., Sunnynale, CA). Peptide sequences were characterized using electrospray ionization mass spectrometry (Bruker Daltonik GmBH, Leipzig, Germany). Analytic results detected by tandem mass spectrometry was submitted to SWISS-PORT bioinformation resource portal (http:// www.expaysy.com) to match the peptide homology.

\section{Proximity ligation assessment of HSP60 and RPTOR interaction}

Interaction between HSP60 and RPTOR in cell cultures was probed using Duolink ${ }^{\circledast}$ PLA Fluorescence Kits
(Sigma-Aldrich). In brief, formaldehyde-fixed cell cultures were blocked using Duolink Blocking Solution and mixed with HSP60 and RPTOR antibodies, and secondary antibody Duolink ${ }^{\circledast}$ PLA probe. Specimens were incubated in a Duolink ligation buffer containing $1 \mathrm{U} / \mu \mathrm{l}$ ligase at $37^{\circ} \mathrm{C}$ for $30 \mathrm{~min}$ and followed by reacting with an amplification solution containing $2 \mathrm{U} / \mathrm{ml}$ polymerase and counterstaining with DAPI. Fluorescent spots in cell cultures were detected using a Zeiss fluorescence microscope, and number of fluorescent spots were counted using image analysis system (Carl Zeiss, Gottingen, Germany).

\section{Lentivirus-shuttled RPTOR gene transfer for glucocorticoid-treated mice}

Animal use protocols were reviewed and approved by IACUC, Kaohsiung Chang Gung Memorial Hospital (IACUC Affidavit No. 2011062101). HEK293 cells were transferred with $1 \mu \mathrm{g}$ pMIF-cGFP-zeo plasmid coding RPTOR along with $1 \mu \mathrm{g}$ pPACKF1 plasmid (System Biosciences, Palo Alto, CA). Culture supernatants were harvested, ultrahigh centrifuged, and titrated using LentiX qRT-PCR Titration Kits (Clontech Laboratories Inc, San Francisco, CA) to prepare $2 \times 10^{9}$ infectious units $/ \mathrm{ml}$ lentivirus particle suspension. Twelve-week-old male FVB mice were anesthetized through inhale isoflurane. Twenty-four mice were evenly divided into two groups and anesthetized to receive $50 \mu \mathrm{l}$ RPTOR or mock lentivirus particle suspension through tail vein injection. One week after administration, mice were intra-peritoneally injected with $10 \mathrm{mg} / \mathrm{kg} /$ day methylprednisolone for 4 weeks. For control group, 12 mice were injected with normal saline only. At the end of experiment, mice were killed to dissect femurs and tibiae for study. Six mice were used for $\mu \mathrm{CT}$ and histological assay, and 6 animals for harvesting bone extract for immunoblotting.

\section{HSP60 transgenic mice}

HSP60 transgenic mice (FVB/NNarl-TgPGK-HSP60; HSP60Tg) were bred, as previously described ${ }^{18}$. Siblings that did not express the construct were designated as wild-type mice. 36 male HSP60Tg and 36 wild-type mice (12-week old) were evenly divided into two groups to receive $10 \mathrm{mg} / \mathrm{kg} /$ day methylprednisolone or vehicle for 4 weeks. After euthanasia, bone tissue was dissected for study. Six mice were used for $\mu \mathrm{CT}$ imaging and histomorphology, six mice for harvesting total RNA in bone tissue, and six animals for isolating bone marrow cells.

\section{$\mu \mathrm{CT}$ assessment of bone mass and microstructure}

Tibiae were X-ray scanned using a Skyscan $1176 \mu \mathrm{CT}$ system (Bruker, Kontich, Belgium), as previously described $^{19}$. BMD $\left(\mathrm{g} / \mathrm{cm}^{3}\right)$, BV/TV (\%), Tb.Th $(\mathrm{mm})$, Tb.N, and Ct.Th $(\mathrm{mm})$ of 200 slices of images with $9 \mu \mathrm{m}$ voxel size 
between growth plate region and midshaft region of proximal tibiae were calculated using SKYSCAN $^{\circledR}$ CTAnalysis software.

\section{Immunohistochemistry and histomorphometry}

Sections of methylacrylate-embedded tibiae blocks were stained using von Kossa reagent. Fluorescent calcein intensity was evaluated using a fluorescence microscope. Three sections of each animal were selected for measuring $\mathrm{BV} / \mathrm{TV}(\%)$ and mineral apposition rate (MAR; $\mu \mathrm{m} /$ day). Immunohistochemical assessment was performed using LC3 antibody and BioGenex Immunohistochemistry Detection Kits (BioGenex, Fremont, CA). TUNEL staining in sections was probed using In Situ TUNEL Detection Kits (Roche Diagnostics $\mathrm{GmbH}$ ). Osteoblasts showing positive immunostaining or TUNEL staining in each field were counted. Three fields in each section, and three sections of each mice were selected for measurement.

\section{Ex vivo osteogenic differentiation of bone marrow mesenchymal cells}

Primary bone marrow mesenchymal cells were isolated, as previously described ${ }^{19}$. In total, $1 \times 10^{5}$ cells/well bone marrow mesenchymal cells were incubated in 24-well plates containing osteogenic medium for 18 days. Mineralized matrix formation was stained using von Kossa staining reagents, and area of mineralized matrix in three random fields in each well was measured. In some experiments, cell cultures were harvested for reverse transcription polymerase chain reaction assessment of osteogenic gene expression.

\section{Statistical analysis}

Experiments of cell culture models were repeated five times. Parametric analysis of variance and Bonferroni post hoc test were utilized to examine whether experimental results among vehicle, mock-treated, and RPTOR-treated groups, as well as the investigations among vehicle- and glucocorticoid-treated HSP60Tg mice and wild-type mice were significant different as the $P$ values were set at $<0.05$.

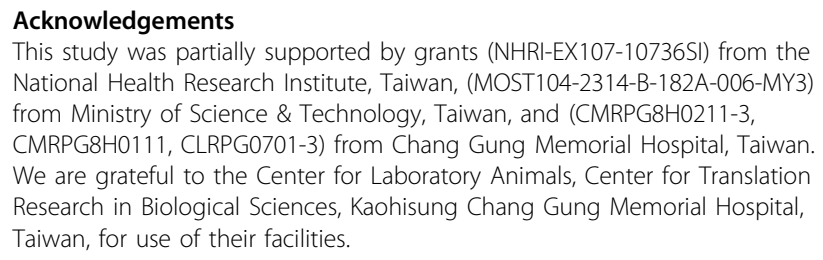
National Health Research Institute, Taiwan, (MOST104-2314-B-182A-006-MY3) from Ministry of Science \& Technology, Taiwan, and (CMRPG8H0211-3, CMRPG8H0111, CLRPG0701-3) from Chang Gung Memorial Hospital, Taiwan. We are grateful to the Center for Laboratory Animals, Center for Translation Research in Biological Sciences, Kaohisung Chang Gung Memorial Hospital, Taiwan, for use of their facilities.

\section{IACUC approval no.}

IACUC affidavit no. 2011062101 by the Institutional Animal Care and Use Committee of Kaohsiung Chang Gung Memorial Hospital.

\section{Author contributions}

Study conception and design: WS Lian, JY Ko, YS Chen, HJ Ke, SL Wu, CW Kuo, FS Wang. Acquisition of data: WS Lian, YS Chen, HJ Ke, SL Wu, CW Kuo. Analysis and interpretation of data: WS Lian, JY Ko, FS Wang. Article drafting and revision: WS Lian, JY Ko, FS Wang.

\section{Author details \\ ${ }^{1}$ Core Laboratory for Phenomics and Diagnostic, Kaohsiung Chang Gung Memorial Hospital, Kaohsiung, Taiwan. ${ }^{2}$ Department of Medical Research, Kaohsiung Chang Gung Memorial Hospital, Kaohsiung, Taiwan. ${ }^{3}$ Department of Orthopedic Surgery, Kaohsiung Chang Gung Memorial Hospital, Kaohsiung, Taiwan. ${ }^{4}$ Graduate Institute of Clinical Medical Science, Chang Gung University College of Medicine, Kaohsiung, Taiwan}

\section{Conflict of interest}

The authors declare that they have no conflict of interest.

\section{Publisher's note}

Springer Nature remains neutral with regard to jurisdictional claims in published maps and institutional affiliations.

Received: 26 April 2018 Revised: 19 August 2018 Accepted: 20 August 2018 Published online: 17 September 2018

\section{References}

1. Rizzoli, R. \& Biver, E. Glucocorticoid-induced osteoporosis: who to treat with what agent. Nat. Rev. Rheumatol. 11, 98-109 (2015).

2. Hartmann, K. et al. Molecular actions of glucocorticoids in cartilage and bone during health, disease, and steroid therapy. Physiol. Rev. 96, 409-447 (2016).

3. Seibel, M. J., Cooper, M. S. \& Zhou, H. Glucocorticoid-induced osteoporosis: mechanisms, management, and future perspectives. Lancet Diabetes Endocrinol. 1, 59-70 (2013).

4. Sato, A. Y., Tu, X. McAndrews, K. A., Plotkin, L. I. \& Bellido, T. Prevention of glucocorticoid induced-apoptosis of osteoblasts and osteocytes by protecting against endoplasmic reticulum (ER) stress in vitro and in vivo in female mice. Bone 73, 60-68 (2015).

5. Zhen, Y. F. et al. P53 dependent mitochondrial permeability transition pore opening is required for dexamethasone-induced death of osteoblasts. J. Cell Physiol. 229, 1475-1483 (2014).

6. Sato, A. Y. et al. Glucocorticoids induce bone and muscle atrophy by tissuespecific mechanisms upstream of E3 ubiquitin ligases. Endocrinology $\mathbf{1 5 8}$ 664-677 (2017)

7. Choi, A. M., Ryter, S. W. \& Levine, B. Autophagy in human health and disease. N. Engl. J. Med. 368, 651-662 (2013).

8. Nollet, M. et al. Autophagy in osteoblasts is involved in mineralization and bone homeostasis. Autophagy 10, 1965-1977 (2014).

9. Vuppalapati, K. K. et al. Targeted deletion of autophagy genes Atg5 or Atg7 in the chondrocytes promotes caspase-dependent cell death and leads to mild growth retardation. J. Bone Miner. Res. 30, 2249-2261 (2015).

10. Cinque, L. et al. FGF signalling regulates bone growth through autophagy. Nature 528, 272-275 (2015).

11. Fitter, S. et al. mTORC1 plays an important role in skeletal development by controlling preosteoblast differentiation. Mol. Cell Biol. 37, e00668-16 (2017).

12. Dai, Q. et al. Inactivation of regulatory-associated protein of mTOR (Raptor)/ mammalian target of rapamycin complex 1 (mTORC1) signaling in osteoclasts increases bone mass by inhibiting osteoclast differentiation in mice. J. Biol. Chem. 292, 196-204 (2017).

13. Nisemblat, S., Yaniv, O., Parnas, A., Frolow, F. \& Azem, A. Crystal structure of the human mitochondrial chaperonin symmetrical football complex. Proc. Natl. Acad. Sci. USA 112, 6044-6049 (2015).

14. Berger, E. et al. Mitochondrial function controls intestinal epithelial stemness and proliferation. Nat. Commun. 7, 13171 (2016).

15. Deng, J. et al. FUS interacts with HSP60 to promote mitochondrial damage. PLoS. Genet. 11, e1005357 (2015).

16. Barone, R. et al. Skeletal muscle heat shock protein 60 increases after endurance training and induces peroxisome proliferator-activated receptor gamma coactivator 1 a1 expression. Sci. Rep. 6, 19781 (2016).

17. Liu, X. et al. Autophagy induced by DAMPs facilitates the inflammation response in lungs undergoing ischemia-reperfusion injury through promoting TRAF6 ubiquitination. Cell Death Differ. 24, 683-693 (2017). 
18. Ko, J. Y., Sun, Y. C., Li, W. C. \& Wang, F. S. Chaperonin 60 regulation of SOX9 ubiquitination mitigates the development of knee osteoarthritis. J. Mol. Med. 94, 755-769 (2016).

19. Wang, F. S. et al. Heat shock protein 60 protects skeletal tissue against glucocorticoid-induced bone mass loss by regulating osteoblast survival. Bone 49, 1080-1089 (2011)

20. Li, J. et al. Dexamethasone shifts bone marrow stromal cells from osteoblasts to adipocytes by C/EBPalpha promoter methylation. Cell Death Dis. 4, e832 (2013).

21. Aluksanasuwan, S., Sueksakit, K., Fong-Ngern, K. \& Thongboonkerd, V. Role of HSP60 (HSPD1) in diabetes-induced renal tubular dysfunction: regulation of intracellular protein aggregation, ATP production, and oxidative stress. FASEB J. 31, 2157-2167 (2017).

22. Marino Gammazza, A. et al. Doxorubicin anti-tumor mechanisms include Hsp60 post-translational modifications leading to the Hsp60/p53 complex dissociation and instauration of replicative senescence. Cancer Lett. 385, 75-86 (2017).

23. Mirigian, L. S. et al. Osteoblast malfunction caused by cell stress response to procollagen misfolding in a2(I)-G610C mouse model of osteogenesis imperfecta. J. Bone Miner. Res. 31, 1608-1616 (2016).

24. Wang, Z. et al. Autophagy mediated CoCrMo particle-induced peri-implant osteolysis by promoting osteoblast apoptosis. Autophagy 11, 2358-2369 (2015).

25. Toscani, D. et al. The proteasome inhibitor bortezomib maintains osteocyte viability in multiple myeloma patients by reducing both apoptosis and autophagy: a new function for proteasome inhibitors. J. Bone Miner. Res. 31, 815-827 (2016)

26. Allaeys, I., Marceau, F. \& Poubelle, P. E. NLRP3 promotes autophagy of urate crystals phagocytized by human osteoblasts. Arthritis Res. Ther. 15, R176 (2013).

27. Wang, A., Midura, R. J., Vasanji, A., Wang, A. J. \& Hascall, V. C. Hyperglycemia diverts dividing osteoblastic precursor cells to an adipogenic pathway and induces synthesis of a hyaluronan matrix that is adhesive for monocytes. J. Biol. Chem. 289, 11410-11420 (2014).

28. Benoit, B. et al. Fibroblast growth factor 19 regulates skeletal muscle mass and ameliorates muscle wasting in mice. Nat. Med. 23, 990-996 (2017).

29. Rohde, K. et al. A Bak-dependent mitochondrial amplification step contributes to Smac mimetic/glucocorticoid-induced necroptosis. Cell Death Differ. 24, 83-97 (2017).
30. van den Beukel, J. C. et al. Cold exposure partially corrects disturbances in lipid metabolism in a male mouse model of glucocorticoid excess. Endocrinology 156, 4115-4128 (2015).

31. Guan, S. S. et al. The pathological role of advanced glycation end productsdownregulated heat shock protein 60 in islet $\beta$-cell hypertrophy and dysfunction. Oncotarget 7, 23072-23087 (2017).

32. Zhou, C. et al. Oncogenic HSP60 regulates mitochondrial oxidative phosphorylation to support Erk1/2 activation during pancreatic cancer cell growth. Cell Death Dis. 9, 161 (2018).

33. Martin, S. K. et al. Brief report: the differential roles of mTORC1 and mTORC2 in mesenchymal stem cell differentiation. Stem Cells 33, 1359-1365 (2015).

34. Chen, J. \& Long, F. mTORC1 signaling controls mammalian skeletal growth through stimulation of protein synthesis. Development 141, 2848-2854 (2014).

35. Joeng, K. S. et al. Osteocyte-specific WNT1 regulates osteoblast function during bone homeostasis. J. Clin. Invest. 127, 2678-2688 (2017).

36. Yamamoto, S. et al. NIP-SNAP-1 and -2 mitochondrial proteins are maintained by heat shock protein 60. Biochem. Biophys. Res. Commun. 483, 917-922 (2017).

37. Wiechmann, K. et al. Mitochondrial chaperonin HSP60 as the apoptosis-related target for myrtucommulone. Cell Chem. Biol. 24, 614-623 (2017).

38. Tang, $\mathrm{H}$. et al. Down-regulation of HSP60 suppresses the proliferation of glioblastoma cells via the ROS/AMPK/mTOR pathway. Sci. Rep. 6, 28388 (2016).

39. Finka, A., Mattoo, R. U. \& Goloubinoff, P. Experimental milestones in the discovery of polypeptide unfolding enzyme. Ann. Rev. Biochem. 85, 715-742 (2016).

40. Dan, H. C. et al. Akt-dependent activation of mTORC1 complex involves phosphorylation of mTOR (mammalian target of rapamycin) by IKB kinase a (IKKa). J. Biol. Chem. 289, 25227-25240 (2014).

41. Parrales, A., López, E., Lee-Rivera, I. \& López-Colomé, A. M. ERK1/2-dependent activation of $\mathrm{mTOR} / \mathrm{mTORC} 1 / \mathrm{p} 70 \mathrm{~S} 6 \mathrm{~K}$ regulates thrombin-induced RPE cell proliferation. Cell Signal. 25, 829-838 (2013).

42. Wang, F. S et al. Histone demethylase UTX counteracts glucocorticoid deregulation of osteogenesis by modulating histone-dependent and -independent pathways. J. Mol. Med. 95, 499-512 (2017). 